\title{
Importance of Plasmonic Scattering for an Optimal Enhancement of Vibrational Absorption in SEIRA with Linear Metallic Antennas
}

\author{
Tomáš Neuman, ${ }^{\dagger}$ Christian Huck, ${ }^{\ddagger}$ Jochen Vogt, ${ }^{\ddagger}$ Frank Neubrech, ${ }^{\S}$ Rainer Hillenbrand, ${ }^{\|, \perp}$ \\ Javier Aizpurua, ${ }^{\dagger}$ and Annemarie Pucci* ${ }^{*}$, \\ ${ }^{\dagger}$ Materials Physics Center, CSIC-UPV/EHU and DIPC, Paseo Manuel de Lardizabal 5, Donostia-San Sebastian 20018, Spain \\ ${ }^{\ddagger}$ Kirchhoff Institute for Physics, Heidelberg University, Im Neuenheimer Feld 227, 69120 Heidelberg, Germany \\ $\S 4$ th Physics Institute, University of Stuttgart, Pfaffenwaldring 57, 70569 Stuttgart, Germany \\ "CIC nanoGUNE and UPV/EHU, Donostia-San Sebastian 20018, Spain \\ ${ }^{\perp}$ IKERBASQUE, Basque Foundation for Science, Bilbao 48011, Spain
}

Supporting Information

ABSTRACT: Surface-enhanced infrared absorption (SEIRA) and surface-enhanced Raman scattering (SERS) represent very effective techniques to detect molecular vibrational fingerprints. These techniques can be improved thanks to the use of plasmonic antennas that produce strong resonant near-fields in their vicinity, enhancing the signal of vibrational samples. Here we study the role of plasmonic absorption and scattering of the hosting antennas in the resulting SEIRA signal. Using numerical simulations of the antenna-sample infrared response, we show that the optimal SEIRA signal measured in transmittance (as extinction) is achieved when the spectral maxima of absorption and scattering of the antennas are of

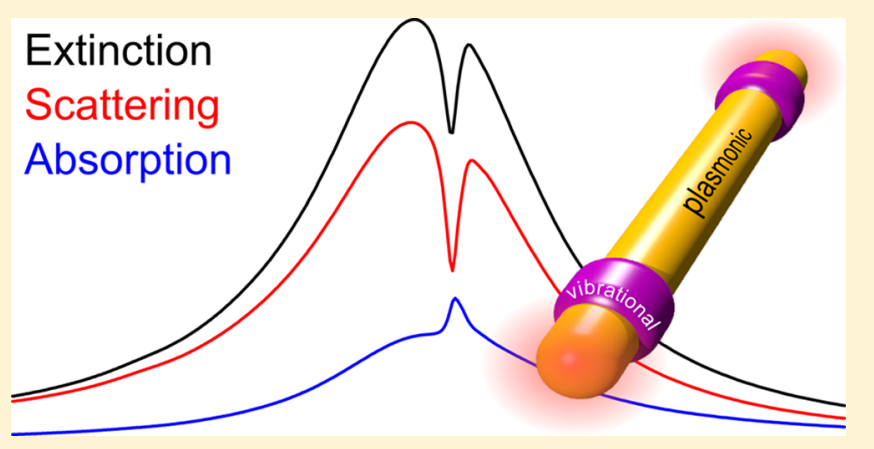
similar magnitude. Paradoxically, when the optimal condition for SEIRA is fulfilled, the decomposition of the signal into the contribution from scattering and from absorption show that the vibrational fingerprint is exclusively a result of the scattering, with no contribution from absorption. Using a simple analytical model for the description of the fundamental resonance of linear nanoantennas made of a Drude-type metal, we provide guidelines for controlling the plasmonic light scattering and light absorption properties, thus showing how the optimal condition for SEIRA can be achieved in practical situations.

\section{INTRODUCTION}

Infrared (IR) vibrational spectroscopy is a powerful tool for the identification of the chemical composition and the molecular geometry via the vibrational fingerprints. However, one big problem hampers the successful application of IR spectroscopy, especially in the case where small amounts of molecules are present. This problem consists in the extremely small IR absorption cross section of molecules which is much smaller than the squared wavelength of the probing IR radiation at the vibrational frequency of the molecule (see Supporting Information). This problem can be overcome by placing the molecule into strongly enhanced electromagnetic near-fields, for example, such of a resonant plasmonic antenna. In this article we will demonstrate that the resonant plasmonic scattering of these hosting antennas is especially relevant for the measurement of enhanced IR vibrational absorption signals.

The first findings on enhanced signals of molecules on metal nanoparticles, ca. 50 years ago, were named "anomalous transmission". This kind of surface-enhanced IR absorption (SEIRA) of adsorbates on metal-nanoparticle aggregates can reach 3 orders of magnitude and can be modeled by effective media theories (EMT) if the particles are small enough. ${ }^{2}$
Usually, the metal nanoparticles in such SEIRA studies have diameters of only a few nanometers, and therefore their light scattering (proportional to the fourth power of the ratio of the particle diameter to the wavelength) almost vanishes in the IR. On the other hand, the optical absorption of these structures even in the IR is strong because the mutual interaction of the nanoparticles broadens and red-shifts their resonances. ${ }^{3}$ The observed vibrational line-shape asymmetry in such SEIRA spectra can thus be correctly simulated within EMT. ${ }^{4}$ Less than 10 years ago, a stronger SEIRA (by almost 6 orders of magnitude) from molecules on micrometer long metal nanowires with their plasmonic resonances matching the vibrational frequencies was detected and identified as a Fano effect with the plasmonic excitation spectrum as the background. ${ }^{5,6}$ The Fano-type coupling between this background and the vibrationally induced dipole is governed by the nearfield strength (apart from the vibrational dipole moment) as it becomes obvious upon detuning of the two excitation spectra

Received: August 27, 2015

Revised: October 28, 2015

Published: October 30, 2015 

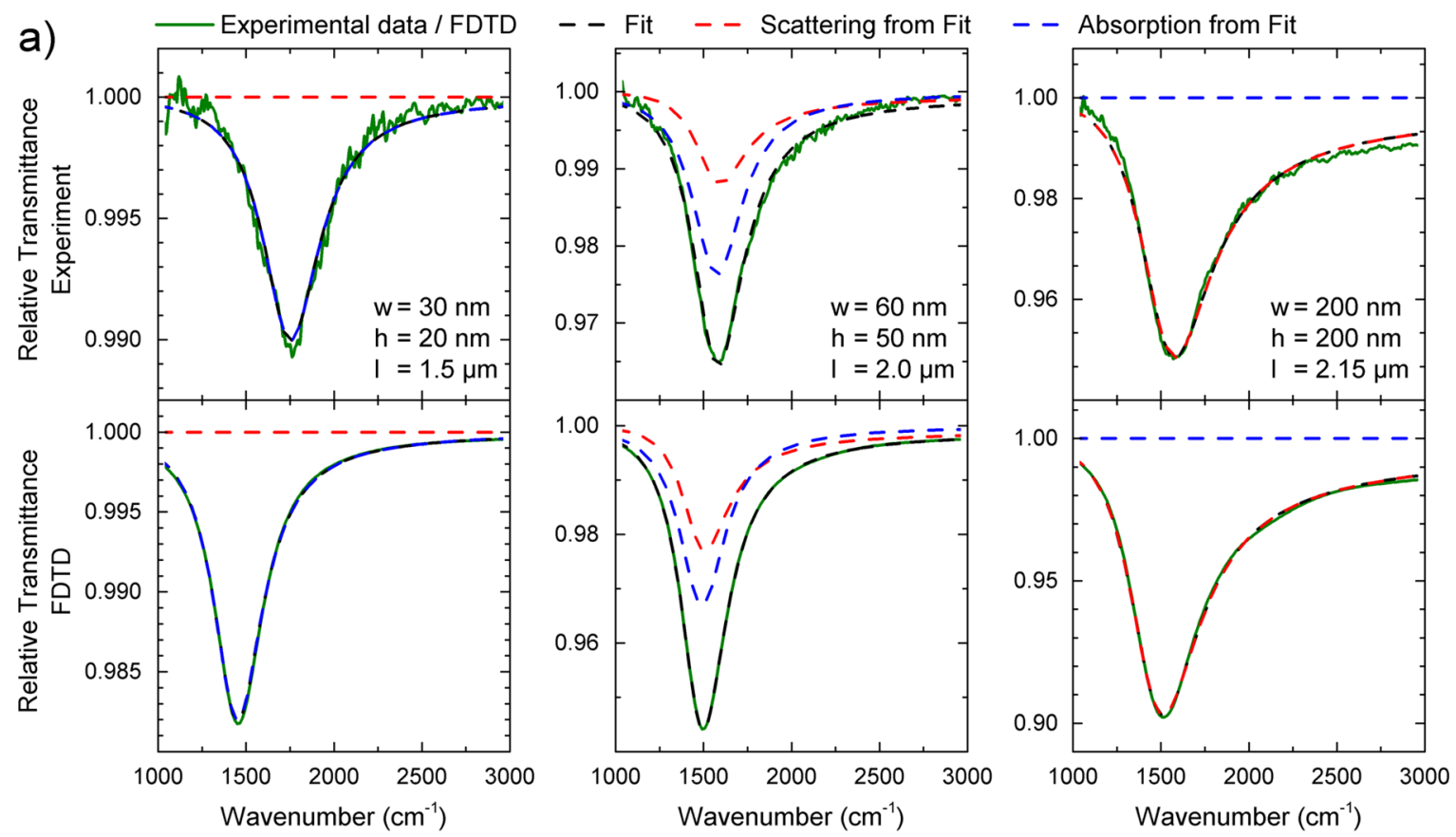

b)

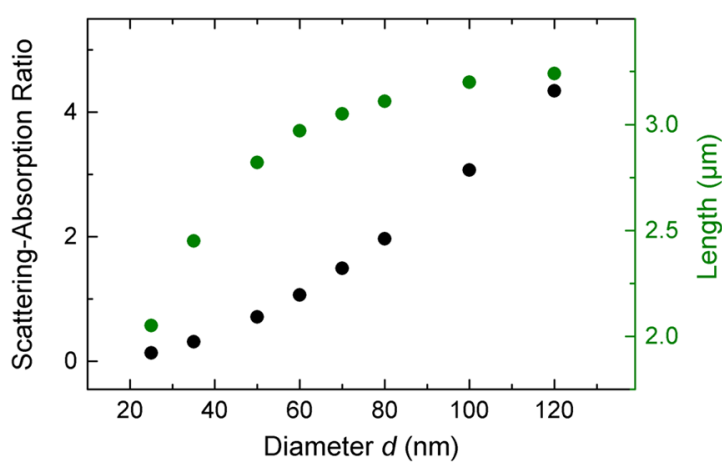

c)

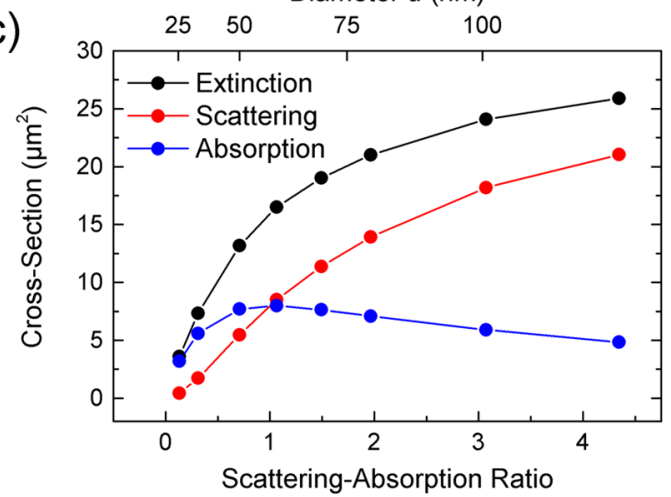

Figure 1. (a) Quasi-static fit to the experimental transmittance (top row) of single nanowires at normal incidence of IR light and to FDTD simulation of such spectra (bottom row) for the same geometries (length $l$, height $h$, and width $w$, given in the panels). The spectra are normalized to the transmittance of the bare $\mathrm{CaF}_{2}$ substrate (refractive index of 1.41). For the experimental spectra in the middle top panel, absorption and scattering could be well separated from the extinction and the fit yields $=236 \pm 22 \mathrm{~cm}^{-1}$. This value is larger than that of the analytical fit to the FDTD simulation (bottom middle panel), where the fit yields $=193 \pm 6 \mathrm{~cm}^{-1}$, a value that corresponds to $\omega_{\tau}$ as obtained from a Drude fit to the textbook data. ${ }^{25,26}$ (b) Relation between the geometrical dimensions of linear plasmonic antennas with diameter $d$ and length $l$ and their scatteringabsorption ratio (extracted from the maximum values of the respective spectra). The ratio was varied via changing the antenna volume and adjusting its aspect ratio, so that the resonance of the antennas was the same for all cases. For further plots, see the Supporting Information. (c) Extinction (black dots), scattering (red dots), and absorption (blue dots) cross sections as a function of the scattering-absorption ratio of the antennas analyzed in (b). Notice the different development of the various maximum cross sections. All the FDTD simulations were done with Palik's data for gold. $^{25}$

(molecules and antennas), which changes the line-shape asymmetry and the signal contrast up to a vanishing signal at complete detuning. Several experiments with various plasmonic objects that are resonant in the IR confirmed the huge vibrational signal enhancement. ${ }^{5-14}$ Following these concepts, various biosensor devices have been demonstrated, as for example, in the detection of biomolecules where the reflection mode of a plasmonic array in contact with a microfluidic cell is used. ${ }^{15}$ The interference behavior of regular arrays of plasmonic nanoparticles can be tuned by means of modifications in the array's lattice constants in a way that specular reflectance is maximized at the plasmonic resonance, which increases sensitivity even more. ${ }^{16}$ Such kind of backscattering geometry works well for sufficiently large plasmonic scattering, a situation that is achieved with plasmonic objects with a size of the order of the wavelength. In this situation, because of the considerable contribution of IR light scattering, EMT cannot provide a correct description of the SEIRA spectra. For example, the phonon polariton excitations near the longitudinal optical (LO) phonon frequency in a film can be observed in normal transmittance when resonant antennas are placed on top of the film, and a polarization of the probing light is parallel to the antenna long axis. In the absence of the antennas or when the light is polarized perpendicular to the antenna axis, the enhancement vanishes. ${ }^{6,17}$ While many facts indicate the particular importance of the plasmonic scattering in SEIRA, it is not yet clear how plasmonic scattering and plasmonic absorption mutually contribute to SEIRA that relies on a signal 


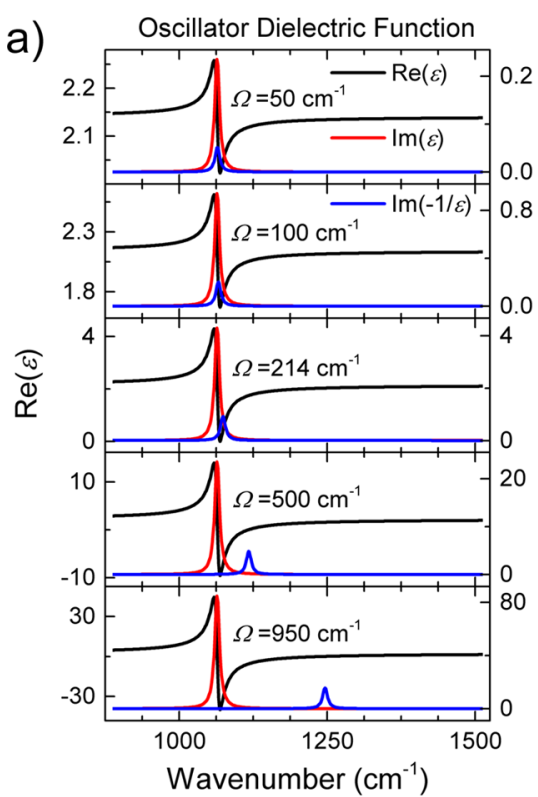

c)

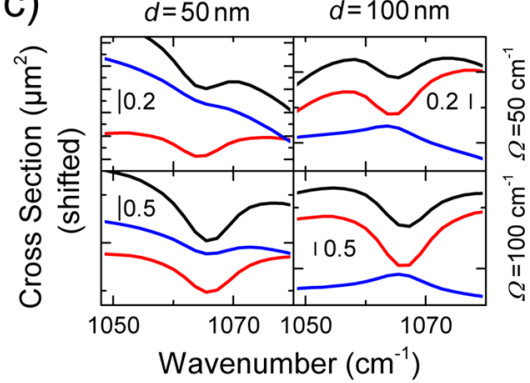

b)

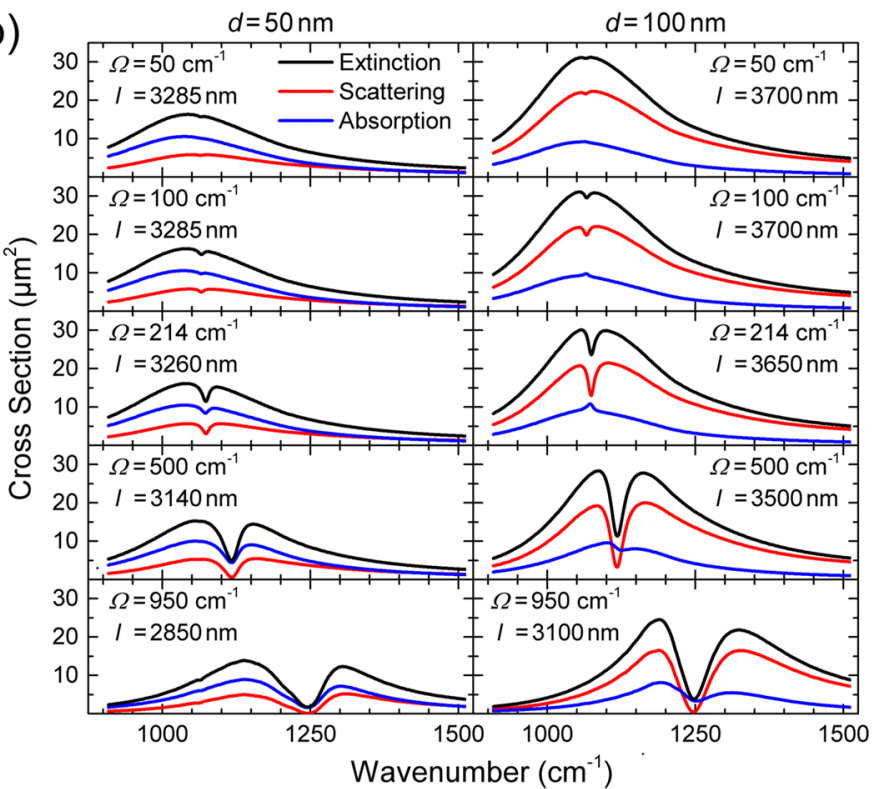

e)

d)

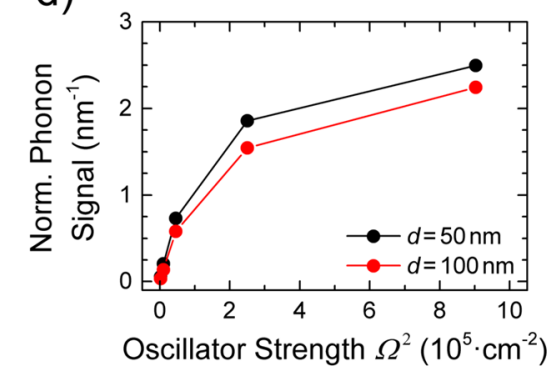

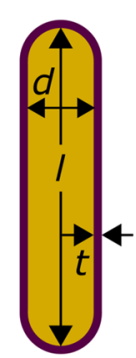

Figure 2. (a) Dielectric response function of the vibrational oscillator (eq 3). Real part (black line), imaginary part (red line), and loss function (blue line) of the dielectric response are shown for different oscillator strengths $\Omega$. Notice that the real part of the oscillator's dielectric function corresponds to a weak oscillator for $\Omega$ below $214 \mathrm{~cm}^{-1}$ (top three panels). (b) FDTD calculations of the cross sections for various strengths $\Omega$ (given in $\mathrm{cm}^{-1}$ ) of the vibrational oscillator (with different lengths $l$ to match the resonance) and two different gold antenna diameters $d$ (simulations with Palik's gold data ${ }^{25}$ ). The vibrational oscillator belongs to a layer of thickness $t=10 \mathrm{~nm}$ covering the whole antenna. (c) A zoom to the vibrational signals for the two smallest $\Omega$. (d) SEIRA signal contrast (normalized to the layer volume that is proportional to $d^{2}$ ) obtained from the baseline corrected extinction cross-section spectrum as a function of oscillator strength. (e) Sketch of the geometry of antenna and sample used in the calculations of the SEIRA signal.

obtained from extinction measurements. Furthermore, the role of the scattering and of the absorption mechanism in the formation of the final extinction signal strongly depends on the material properties of the hosting plasmonic particles as well as on their geometry.

In order to investigate the role of plasmonic scattering and absorption in SEIRA, we consider the representative case of single linear gold nanoantennas with micrometer length and a high aspect ratio of about 10 (or arrays of such antennas with large enough spacings that prevent the coupling between the antennas ${ }^{18}$ ) under an illumination with light that is polarized parallel to the antenna axis. We first examine the properties of scattering and absorption of bare IR resonant nanoantennas and introduce an analytical model for the plasmonic absorption and extinction cross sections which helps to analyze experimental transmittance spectra and to understand the role of intrinsic electronic damping in both plasmonic light scattering and absorption. In the next step, the enhanced signals from IR-active vibrational oscillators of isotropic dielectric layers are inspected. For various vibrational oscillator strengths we analyze the vibrationally induced spectral changes (that we call "contrast") in plasmonic absorption, plasmonic scattering, and plasmonic extinction. Then the role of the spatial distribution of the vibrational oscillators with respect to the antenna is studied by positioning a dielectric nanoparticle characterized by a strong vibrational oscillator along the antenna. We will show that the spectral signature of SEIRA of this oscillator in the plasmonic scattering spectrum dominates under various conditions of plasmonic coupling, whereas the SEIRA signal on the plasmonic absorption spectrum may vanish. These findings, based on finite-difference time domain (FDTD) simulations, corroborate a consistent, very simple, and practical rule for designing the optimal plasmonic hosting objects for SEIRA applications. The implementation of this rule requires a careful inspection of the scattering and the absorption cross section of the plasmonic antenna.

\section{EXPERIMENTAL AND SIMULATION METHODS}

Sample Preparation. The single nanoantennas (for which the measurements are shown in Figure 1a) were fabricated by means of standard electron beam lithography (EBL). ${ }^{17}$ The antennas were prepared on $\mathrm{CaF}_{2}(100)$ substrate. A chromium adhesion layer $(2 \mathrm{~nm})$ and gold (as layer of height $h$ ) were evaporated on the patterned photoresist. Each antenna was designed in cuboid shape with the dimensions length $l$, width $w$, 
and height $h$. The values for $l, w$, and $h$ given in Figure 1 a are the preset parameters of the preparation. Usually, they are checked with scanning electron microscopy (SEM) or atomic force microscopy (AFM), and errors in the production can be evaluated. For high quality EBL and precise layer deposition, deviations from the preset parameters are less than $10 \mathrm{~nm}$. In the Supporting Information we show a SEM picture of a typical EBL made IR resonant gold antenna.

IR Measurements. Microscopic IR spectra of single nanoantennas (Figure 1a) were acquired using radiation from the synchrotron light source ANKA. The relative transmittance measurements at normal incidence of light (polarized parallel to the long antenna axis) were conducted with a Bruker IFS $66 \mathrm{v} / \mathrm{S}$ spectrometer connected to an IRscope II microscope equipped with a liquid-nitrogen-cooled mercury cadmium telluride detector. With a circular aperture of $14.7 \mu \mathrm{m}$ in diameter and a resolution of $8 \mathrm{~cm}^{-1}$, at least 500 scans were recorded for each spectrum. In order to locate the antennas, the spot of the highest plasmonic response was determined by performing systematic area mapping measurements of their surroundings.

Numerical Simulations. Finite-difference time-domain simulations (FDTD Solutions, Lumerical Solutions, Inc.) were performed to calculate the scattering and absorption cross sections as well as near-field and phase distributions around the antennas (for all data except Figure 1a). A cylindrically symmetrical gold antenna with a diameter $d$ between 20 and $120 \mathrm{~nm}$ (see Figure $1 \mathrm{~b}$ ), length $l$ between 2 and $3.3 \mu \mathrm{m}$, and semispherical apexes (curvature radius $d / 2$ ) (see Figure 2e) was implemented in a large enough simulation cell surrounded by perfectly matched boundary conditions. To account for the small antenna diameter and the thin layer, a mesh size down to $1 \mathrm{~nm}$ was used around the antenna apex and around the dielectric nanoparticle. The mesh around the dielectric nanoparticle was refined to $0.5 \mathrm{~nm}$ and around the semispherical apexes of the antenna, if covered by the dielectric layer, to $1.5 \mathrm{~nm}$. The antenna was illuminated using a broadband $\left(800-2000 \mathrm{~cm}^{-1}\right)$ plane wave polarized along the long antenna axis. A sufficiently long time evolution was allowed as to get full convergence of the response. The absorption and scattering cross sections were calculated by considering the net power flowing inward and outward, respectively, through the respective evaluation surface embedding the antenna. The SEIRA signal size (contrast) is evaluated as a difference between the maximum and the minimum of the baseline corrected cross-section spectrum, ${ }^{5,11,14,19,20}$ using the smoothing algorithm proposed by Eilers. ${ }^{21}$ In the SEIRA simulations, the length and the diameter respectively of the antenna are chosen to lead to a match of the plasmonic resonance and the vibrational spectral feature, which is the basic condition for a high SEIRA signal. ${ }^{5,6,19,20}$ The values of $l$ and $d$ are given in the figures. The given length $l$ is the total length including the caps.

\section{RESULTS AND DISCUSSION}

Scattering, Absorption, and Extinction of Plasmonic Antennas. The IR plasmonic extinction of resonances of micron long antennas contains an important contribution from resonant light scattering, in contrast to the metal nanoparticles that are resonant in the visible (VIS). In linear, rod-shaped IR antennas, for instance, this contribution becomes even larger (compared to absorption) for larger diameters $d$ (or larger height $h$ and width $w$ ). As a surprisingly good approximation, the extinction of the longitudinal nanowire resonances can be nicely described within the radiation-corrected quasi-static approximation. The quasi-static approximation in principle is appropriate for an incident field that is nearly uniform across the object and for particles that are smaller than about $1 \%$ of the wavelength, but nevertheless, it is frequently used for firstorder estimates of resonances for substantially larger objects, as mentioned in the textbook by D. Sarid and W. A. Challener, ${ }^{22}$ for example. In the radiation-corrected quasi-static approximation for nanoantennas from a Drude-type metal like gold, silver, and copper (and other metals with free electrons only from $\mathrm{s}$ and $\mathrm{p}$ states) with an intrinsic electronic relaxation rate, $\omega_{\tau}$, that is smaller than the frequency of IR light, $\omega\left(\omega_{\tau} \ll \omega\right)$, the analytical relations for the scattering cross section

$$
\sigma_{\text {sca }}=\frac{6 \pi c^{2}}{n_{\text {host }}^{2}} T \frac{\omega^{4} T}{\left[\left(\omega_{\text {res }}^{2}-\omega^{2}\right)^{2}+\omega^{2}\left(\omega_{\tau}+\omega^{2} T\right)^{2}\right]}
$$

and the absorption cross section

$$
\sigma_{\mathrm{abs}}=\frac{6 \pi c^{2}}{n_{\text {host }}{ }^{2}} T \frac{\omega^{2} \omega_{\tau}}{\left[\left(\omega_{\text {res }}^{2}-\omega^{2}\right)^{2}+\omega^{2}\left(\omega_{\tau}+\omega^{2} T\right)^{2}\right]}
$$

differ in their frequency-dependent spectral shape only due to the differences in the numerators. $T=V R n_{\text {host }} \omega_{\mathrm{p}}^{2}$ is the Larmor-time parameter (with the vacuum velocity $c$ of light) depending on the plasma frequency of the metal $\omega_{\mathrm{p}}$, the volume $V$ (where the electrons are excited), the refractive index $n_{\text {host }}$ of the surrounding host medium, and the local-field ratio $R^{23}$ For a spheroid in the quasi-static approximation, $R=\varepsilon_{\mathrm{s}} /\left[\varepsilon_{\mathrm{s}}\right.$ $\left.+F\left(\varepsilon_{\infty}-\varepsilon_{s}\right)\right]$ with $F$ as the depolarization factor. ${ }^{24}$ For a sphere, for example, $R=3 \varepsilon_{\mathrm{s}} /\left(\varepsilon_{\infty}+2 \varepsilon_{\mathrm{s}}\right)$ for an infinitely long needle $R=1$, which is a reasonable approximation also for nanowires. Radiation damping, due to the charges with a timedependent acceleration, is included in terms of a damping rate, $\omega_{\mathrm{rad}}=\omega^{2} T$, in the denominators of the quasi-static expressions, $\sigma_{\text {abs }}$ and $\sigma_{\text {scat. }}$. Typical values of $\omega_{\text {rad }}=\omega_{\text {res }}{ }^{2} T$ for IR nanoantennas in gold (with $h$ and $w$ approximately $50 \mathrm{~nm}$, $\left.n_{\text {host }} \geq 1\right)$ are of the order of the relaxation rate and thus are not negligible. An extreme case of intrinsically damped antennas are metal nanoparticles with geometric dimensions much shorter than the distance traveled by a bulk electron at the Fermi level within one period of the IR field. For these particles, radiation damping is much smaller than the intrinsic electronic damping that is further increased due to the additional electronic scattering on the antenna surface with respect to the bulk electronic damping observed for larger antennas. Clearly, the balance between both electronic and radiative damping ultimately determines the performance of absorption and scattering in the extinction signal. Figure la displays the relative transmittance due to the plasmonic extinction of nanoantennas (top row experimental results and lower row FDTD calculation) together with the corresponding fits to the function $\sigma_{\text {ext }}=\sigma_{\text {abs }}+\sigma_{\text {sca }}$ (the sum of eqs 1 and 2). The fit parameters are $\omega_{\tau}$, the resonance frequency $\omega_{\text {res }}$, and the parameters $T$ and $n_{\text {host }}$. The results show the strong effect of the antenna size (increasing from left to right panels) on the ratio between absorption (blue dashed lines) and scattering (red dashed lines). Remarkably, in the range of about $50 \mathrm{~nm}$ of transverse antenna dimensions, IR absorption and scattering are of similar importance in the plasmonic extinction of gold nanowires. Because of the excellent agreement of the fit both for the experiments and the theoretical calculations, this approximation seems to be a reasonable description for the rather thin 
antennas, although the antenna length reaches ca. $20-30 \%$ of the wavelength. Therefore, we can further use eqs 1 and 2 to control the mutual behavior of scattering and absorption in an antenna.

We can consider an idealized case of vanishing intrinsic damping, which establishes an ideal scattering cross section $\sigma_{\text {sca }}\left(\omega_{\text {res }}, \omega_{\tau}=0\right)=6 \pi c^{2} /\left(n_{\text {host }} \omega_{\text {res }}\right)^{2}$ with $\sigma_{\text {abs }}=0$. In such an idealized situation, the signal in SEIRA would be mediated exclusively by plasmonic scattering. In the opposite situation, for small antenna volumes (with $T \rightarrow 0$ ) only plasmonic absorption would occur.

It has been proven by previous studies that strong SEIRA signals can be obtained only when the resonance of the hosting antenna matches the vibrational resonance of the sample. ${ }^{5,19,20}$ Here we point out that the ratio between the antenna's scattering losses and intrinsic losses is an important design parameter to consider for the hosting antennas which can be tuned to optimize the SEIRA performance. In order to prove this, we present results for a series of antennas which have the same resonance frequency but different volume and hence different proportion between the maximum values of their scattering and absorption cross sections (scattering-absorption ratio). The map of this scattering-absorption ratio versus the geometrical dimensions of the antennas is plotted in Figure $1 \mathrm{~b}$. The plasmonic scattering increases with respect to the plasmonic absorption for larger antennas. A saturation of the antenna length can be observed in the figure due to the requirement to keep fixed the antenna resonance.

In Figure 1c, we plot the values of the FDTD results for the maxima of the antenna extinction, scattering, and absorption cross sections as a function of the scattering-absorption ratio. The maximum value of the antenna's extinction cross-section spectrum grows monotonously due to the increasing contribution of the scattering cross section. On the other hand, the maximum of the plasmonic absorption cross section shows an optimal value (and so the extinction cross section if normalized to the geometrical cross section; see Supporting Information Figure S2b) when the antenna's scattering and absorption are equal. This phenomenon is well-known in the theory of resonators where this is referred to as the critical coupling condition, characterized by the equality of the resonator intrinsic damping and the coupling (losses) to the radiation fields (according to eqs 1 and 2, $\omega_{\text {res }}{ }^{2} T=\omega_{\tau}$ which is equivalent to $\sigma_{\mathrm{abs}}=\sigma_{\mathrm{sca}}$ ). For this condition, the maximum near-field enhancement is obtained, as it was shown, for example, by Seok et al. in terms of quality factors. ${ }^{27}$ In the following, we will demonstrate that the concept of absorption and scattering cross sections matching is also relevant for SEIRA.

Influence of the Vibrational Oscillator Strength on the SEIRA Signal. To elucidate in SEIRA the role of plasmonic scattering and absorption coupled to vibrational samples, we investigate the dependence of the signal on (i) the strength of the vibrational oscillator, (ii) the local near-field enhancement at the position of the vibrational sample, (iii) the amount of vibrational oscillators, and (iv) the size of the hosting plasmonic antenna. In all these situations the plasmonic resonance will be tuned to match the molecular vibrations. The sample vibrational oscillator is described by means of a single Lorentzian oscillator model. For the Lorentzian model used here, the frequency-dependent dielectric function is

$$
\varepsilon_{\text {Lorentz }}(\omega)=\varepsilon_{\infty}+\frac{\Omega^{2}}{\omega_{\mathrm{TO}}{ }^{2}-\omega^{2}-i \gamma \omega}
$$

where a background of $\varepsilon_{\infty}=2.14$ corresponds to vitreous $\mathrm{SiO}_{2}$ material and the oscillator strength $\Omega=950 \mathrm{~cm}^{-1}$ and the damping $\gamma=10 \mathrm{~cm}^{-1}$ correspond to data for the strongest $\mathrm{Si}-$ $\mathrm{O}-\mathrm{Si}$ stretching oscillator, with the TO phonon frequency $\omega_{\mathrm{TO}}$ $=1064 \mathrm{~cm}^{-1}$ (given in photon wavenumbers). ${ }^{28}$ The maximum of the negative imaginary part of the inverse dielectric function $(\operatorname{Im}[-1 / \varepsilon]$, the energy-loss function) defines the spectral peak position, $\omega_{\mathrm{LO}}$, of the $\mathrm{LO}$ phonon, as obtained in inelastic electron scattering and in grazing-incidence reflectance of $\mathrm{p}$ polarized light of ultrathin layers on metals, via the Berreman effect. $^{29}$ As shown already by Fuchs and Kliewer, ${ }^{30}$ such longwavelength excitations in ultrathin layers are due to the vibrational dipoles perpendicular to the layer. For example, Senet et al. ${ }^{31}$ have shown that this kind of excitation represents a thin-film phonon polariton with the polariton wave vector parallel to the film. With decreasing oscillator strength, the position of such vibrational excitation shifts toward the TO frequency. This effect can be clearly identified in Figure 2a where the values for the real and imaginary parts of the dielectric function of the vibrational oscillators is shown together with their loss function, for different oscillator strengths. To study the role of the oscillator strength in the SEIRA signal, we calculate the spectral response of a system composed of a plasmonic antenna surrounded by a dielectric layer (with a dielectric function according to eq 3) (see Figure $2 b)$. Figure $2 b$ compares SEIRA spectra of weaker vibrational oscillators (which do not show a negative real part of their dielectric function) to SEIRA spectra of stronger vibrational oscillators. Weak oscillators are typical of most organic compounds and biomolecules while strong oscillators are typical of optical phonons in ionic crystals. We investigate the role of the oscillator strength $\Omega$ (proportional to the vibrational dipole moment) for layers with the same thickness $t$ on nanoantennas with two different diameter $d$, as a way to modify the ratio between the antenna scattering and absorption cross sections, and thus to produce different regime of antennasample coupling. The antenna lengths are tuned so that there is a good match of the plasmonic resonance to the maximum of the vibrational energy-loss function, $\operatorname{Im}\left[-1 / \varepsilon_{\text {Lorentz }}\right]$. The oscillator strength $\Omega$ and thus the coupling regime are varied from the very weak case (top of Figure $2 b, c$ ) to a strong one (where the real part of $\varepsilon_{\text {Lorentz }}(\omega)$ is negative). Increasing the oscillator strength leads to the formation of characteristic spectral features on the plasmonic spectra, which result in the appearance of a transparency window for a very strong oscillator (bottom graphs in Figure $2 \mathrm{~b}$ ). Importantly, we can observe how the signal in scattering and absorption can show substantially different features. Whereas scattering shows a pronounced dip for all oscillator strengths (red lines), the spectral features in absorption (blue lines) depend on the particular properties of the antenna host and sample. For large antennas (scattering > absorption) and weak oscillator strengths (top-right panels of Figure $2 b$ and corresponding zoom-in plots), positive bumps (peaks) appear in absorption, compensating for the negative dips of scattering in the final extinction signal.

The effect of these vibrational absorption peaks is indeed to diminish the larger scattering dip-like vibrational signal measurable in extinction. This behavior is related to the ratio between the intrinsic losses and the radiation scattering. ${ }^{32,33}$ 

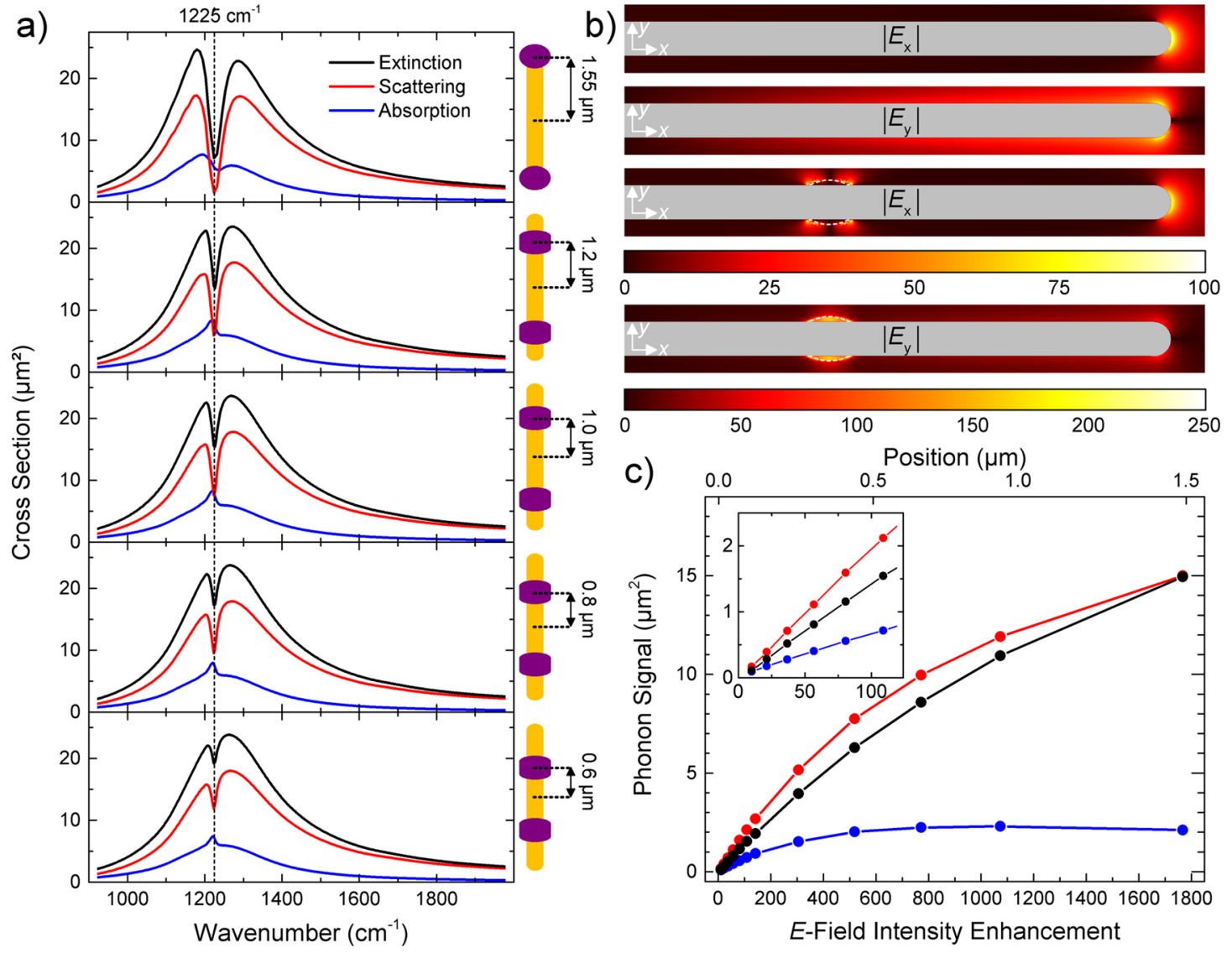

Figure 3. (a) FDTD results of the scattering, absorption, and extinction cross sections for $\mathrm{SiO}_{2}$-like particles of $15 \mathrm{~nm}$ thickness at various sites on the antenna (Palik's gold, ${ }^{25} l=3200 \mathrm{~nm}, d=100 \mathrm{~nm}$ ) as schematically depicted to the right of each panel. (b) Top panels: near-field distribution at resonance $\left(1225 \mathrm{~cm}^{-1}\right)$ shown for the half of a bare antenna for the $x$ - and $y$-component of the electric field under illumination polarized parallel to the antenna axis. Bottom panels: same situation as in the top panels with the $\mathrm{SiO}_{2}$-like particle located at a position of $0.6 \mu \mathrm{m}$ from the antenna center (bottom panel in (a)). (c) Phonon signal (dip size) in absorption, scattering, and extinction cross section for the different particle positions versus the near-field intensity taken $7.5 \mathrm{~nm}$ above the bare antenna surface. Except the antenna apex, the phonon signals are mainly due to the field enhancement of the $y$-component. (Values for the cap at the antenna apex for which the near-field enhancement concerns the $x$-direction are shown in Figure S5 of the Supporting Information.) The inset shows a zoomed area of low field enhancement, where the signal is a linear function of local field intensity enhancement.

Only if $\omega_{\text {res }}^{2} T>\omega_{\tau}$ the antenna absorption shows vibrational absorption peaks that decrease the total dip-like signal in the extinction. As we will see later, the matching of the plasmonic antenna radiation and absorption losses proves to be critically important for the optimization of the SEIRA signal on top of the plasmonic spectrum (i.e., the SEIRA contrast).

SEIRA Signal in Relation to the Position of Vibrational Oscillators on the Plasmonic Antenna. We explore now another relevant aspect that varies the interaction strength between the hosting plasmonic antenna and the sample: the strength of the antenna's local near-field at the position of vibrational oscillators. In order to study this aspect, we change the spatial location of $\mathrm{SiO}_{2}$-like dielectric nanoparticles on the plasmonic antenna, as depicted schematically to the right of panels in Figure 3a. We first calculate SEIRA for a nanoparticle of " $\mathrm{SiO}_{2}$ " located on different sites of the linear plasmonic antenna under an illumination that is polarized parallel to the antenna axis (for simplicity, we always consider a symmetrically positioned pair). The near-field enhancement rises along the antenna axis, from negligible values close to the antenna center to relatively large values close to the antenna apex, establishing a so-called hot spot (see top panel distribution of local fields in Figure $3 \mathrm{~b}$ ). By shifting the sample particle along the antenna axis, we therefore change the strength of the acting near-field and so the coupling of the sample with the plasmonic antenna. We assume the particle to be a spherical cap when located on the antenna apex, and a laterally flattened ring (geometries described in more detail in Figure S3), when it is located along the antenna, as schematically depicted in Figure 3a. The thickness of the particle is $15 \mathrm{~nm}$. The particle itself shows a small extinction, which is almost to $100 \%$ derived from the absorption contribution (see Figure S4). We should mention that the IR light scattering by the tiny volume of the dielectric layer (or cap or ring) itself is several orders of magnitude lower than its vibrational IR absorption. This absorption though is still very small compared to that of the antenna. In other words, without the antenna, the vibrational excitation of the particle would be spectrally unresolvable (practically invisible). The reason to have a strong SEIRA signal is thus due to the interplay of the plasmonic excitation (scattering and absorption) and the vibrational excitation (mostly absorption).

When the vibrational particle is placed closer to the plasmonic antenna's hot spot (apex), as shown in Figure 3a (top panel), the vibrational signal gets dramatically enhanced compared to intermediate positions along the antenna (position marked with a dashed line). There appears a Fano 

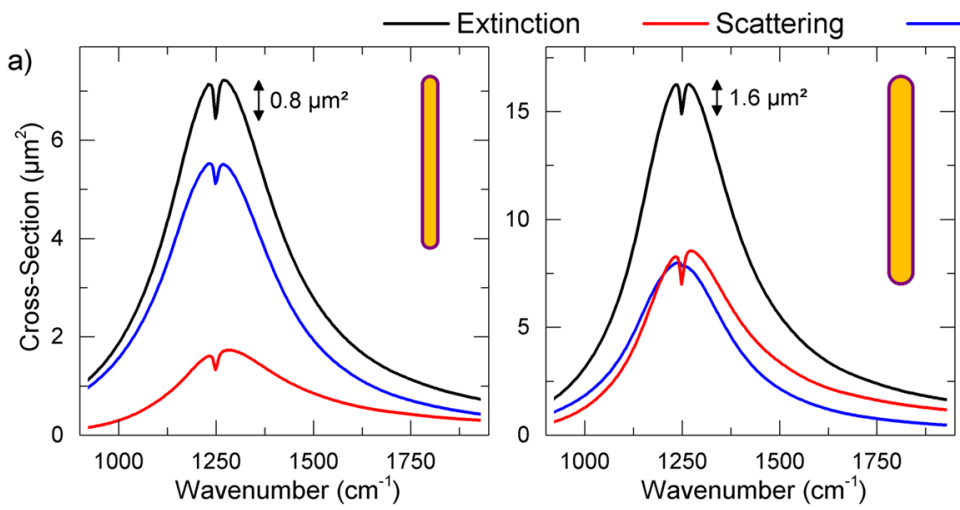

Absorption
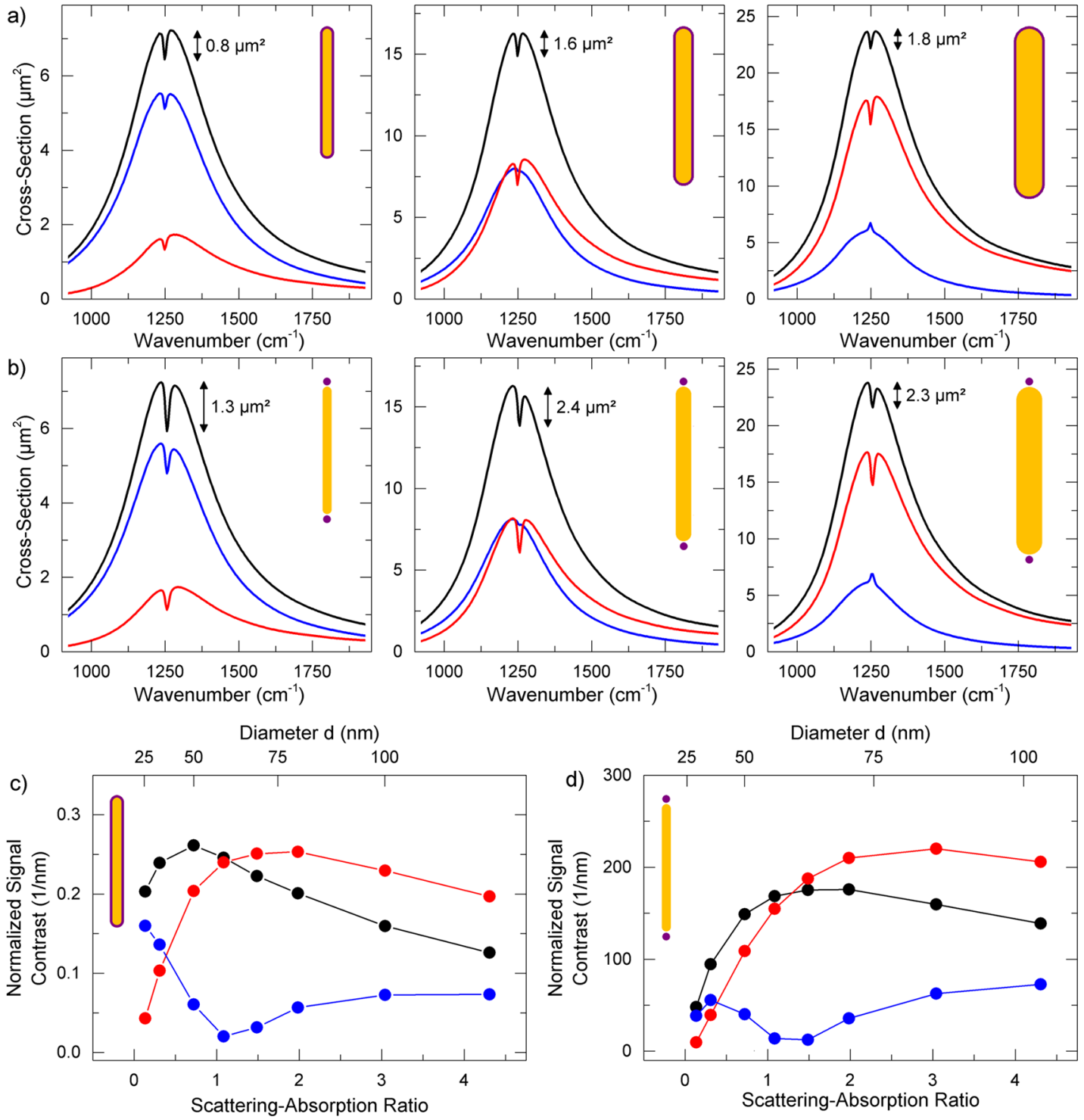

Figure 4. (a, b) Absorption, scattering, and extinction cross-section spectra of a dominantly absorbing antenna (left, $d=35 \mathrm{~nm}, l=2.45 \mu \mathrm{m}$ ), a larger antenna with scattering approximately equal to absorption (middle, $d=60 \mathrm{~nm}, l=2.97 \mu \mathrm{m}$ ), and an antenna that dominantly scatters (right, $d=100$ $\mathrm{nm}, l=3.2 \mu \mathrm{m}$ ). The resonance frequency of the hosting antennas is kept at approximately $1250 \mathrm{~cm}^{-1}$. (a) The sample is a dielectric layer homogeneously covering the antenna surface (thickness $t=10 \mathrm{~nm}$ ). (b) Spectra with a pair of dielectric spheres that are symmetrically placed close to the antenna apexes (the hot spots); sample sphere diameter $d=30 \mathrm{~nm}$, antenna-sphere gap $g=5 \mathrm{~nm}$. (c, d) Vibrational signal contrast in the absorption, scattering, and extinction cross-section spectra (normalized to the volume of the dielectric layer) versus the ratio between plasmonic scattering and absorption (see Figure $1 \mathrm{~b}, \mathrm{c}$ ) for fixed plasmonic resonance frequency and varying volume of the hosting antenna. The dielectric material is modeled by a Lorentzian dielectric function according to eq 3 with $\Omega=1033 \mathrm{~cm}^{-1}, \omega_{\mathrm{TO}}=1157 \mathrm{~cm}^{-1}, \varepsilon_{\infty}=2.14$, and $\gamma=10 \mathrm{~cm}^{-1}$ for the spheres and $\Omega=56 \mathrm{~cm}^{-1}, \omega_{\text {TO }}=1248 \mathrm{~cm}^{-1}, \varepsilon_{\infty}=1$, and $\gamma=10 \mathrm{~cm}^{-1}$ for the layer.

dip in all the antenna scattering spectra (red lines, which decreases with distance from the antenna apex), but the clearly smaller vibrational feature in the plasmonic absorption spectrum (blue lines) changes only slowly in magnitude. Furthermore, the vibrational feature in the plasmonic absorption changes its line shape from a slightly asymmetric dip to an almost symmetric positive peak for a position near the antenna center where the near-field enhancement is low. Such transition from the negative dip to the positive peak happens despite the fact that the vibrational sample (i.e., the dielectric particle) is coupled to the same antenna in all cases.

Because the bare hosting plasmonic antenna has a stronger scattering contribution to the extinction than that from absorption, one would expect only the formation of the positive peak on top of the absorption spectrum, as predicted by the impedance-matching theory. ${ }^{33}$ Here we observe the transition from the positive absorption bump when the sample is close to the antenna center to the negative dip when the dielectric particle is close to the antenna apex as a mere effect of the transition from a weak coupling regime toward a strong coupling regime. In the strong coupling regime (top panel), the well-known transparency window is gradually formed in all of the spectra: scattering, absorption, and extinction. It should be also noticed that the positive peak in the absorption signal when the sample is at the antenna center lowers the total vibrational signal extinction, and thus such a situation is not 
beneficial for sensing. This situation is fully analogous to the case of a weak vibrational oscillator on strongly scattering antennas discussed above (see Figure $2 \mathrm{~b}, \mathrm{c}$ for $d=100 \mathrm{~nm}$ ).

Figure $3 \mathrm{~b}$ displays the near-field distributions at the spectral dip for two electric field components: in the $x$-direction along the antenna (top panel) and in the $y$-direction perpendicular to the antenna (second top panel) for a bare antenna and one with a particle at a position of $0.6 \mu \mathrm{m}$ away from the antenna center. In the absence of the sample particle, the distribution of near-fields in the vicinity of the antenna clearly shows that the field is perpendicular to the antenna surface all over the antenna. When the particle is present (bottom panels of Figure $3 \mathrm{~b})$, this perpendicular component of the field gets captured into the particle, selecting a particular phononic mode of excitation.

The connection between the phonon signal (spectral dip feature) and the electric field intensity enhancement is not straightforward, as shown in Figure $3 \mathrm{c}$, where we plot the SEIRA contrast for scattering (red line), absorption (blue line), and extinction (black line) as obtained from our FDTD calculations, assuming the field enhancement at the particle positions. For positions of the particle close to the antenna center (low field enhancement and thus weak coupling) a linear relation of the signal to the field intensity enhancement can be observed (see inset in Figure 3c). This dependency is consistent with a mechanism of double scattering by the antenna that accounts for the interaction with the vibrational excitations of the dielectric nanoparticle, affecting both scattering and absorption. ${ }^{34}$ In such a case (weak coupling), it can be shown that the modification of the signals giving the SEIRA dips depends linearly on the local field intensity enhancement, which will be detailed elsewhere. For a particle with weak oscillators, the linearity to the near-field intensity was already experimentally verified. ${ }^{35}$ In our simulations, however, when the sample-antenna interaction becomes strong (i.e., when the sample is positioned closer to the antenna apex, or even at the apex), the multiple scattering between the sample and the antenna will lead to a complex evolution of the vibrational signal with the near-field intensity which can produce peculiar behaviors of absorption, scattering, and extinction (right-hand side of Figure 3c). Interestingly, we have demonstrated that the transition between the weak coupling regime to the strong coupling one can be systematically tuned by placing the vibrational sample along the antenna into places of different near-field enhancement.

Importance of the Scattering-Absorption Ratio of the Hosting Plasmonic Antenna for the SEIRA Signal. We finally analyze the importance of scattering in the SEIRA signal from vibrational excitations of dielectric samples hosted on canonical antennas with various ratios between antenna absorption and scattering, as those in Figure 1. We perform simulations of two types of samples; first, we assume a dielectric nanolayer homogeneously covering the antennas, and second, we consider the situation of spherical dielectric nanoparticles in the proximity of the antenna apexes (see insets in Figures $4 \mathrm{a}$ and $4 \mathrm{~b}$, respectively). The thickness of the layer is $10 \mathrm{~nm}$, and the diameter of the particle is $30 \mathrm{~nm}$. Such a nanoparticle can be, for example, a single biological object or a single crystal of a phononic material which can be positioned by a suitable technique close to the hosting antenna surface. Another configuration that adopts a similar arrangement occurs in scattering-type near-field infrared microscopy, where a probing tip, acting as the hosting antenna, probes the vibrational fingerprints of a target sample. ${ }^{36}$ For the layered sample studied here, the parameters of the Lorentzian oscillator characterizing its dielectric function (see eq 3) are chosen so that the layer only weakly interacts with the antenna. Additionally, in order to keep the SEIRA features at the plasmonic resonance frequency, the vibrational oscillator parameters (see caption of Figure 4) of the layer are tuned so that the vibrational mode coincides with the antenna plasmonic resonance, and any effect of the background dielectric function on the plasmonic resonance position is suppressed. The particles in our simulations are two identical spheres symmetrically placed near the hosting antenna apexes $5 \mathrm{~nm}$ away from the antenna surface. The sphere's oscillator parameters (see caption of Figure 4) together with the imposed boundary conditions of the spherical samples provide a good match between the sample's vibrational resonance and that of the hosting antenna. As we will see, for the chosen $\Omega$ corresponding to a strong vibrational (i.e., a phononic) oscillator, even these relatively small spherical samples produce a clear spectral feature on top of the wide antenna resonance. In Figure 4, we plot the cross-section spectra of the antennas modified by presence of the vibrational layer (Figure 4a) and the vibrational sphere pair (Figure 4b). Three panels show the results for an antenna that is dominantly absorbing (thin antenna, left), an antenna that is equally absorbing and scattering (middle), and an antenna that is dominantly scattering (thick antenna, right). The spectral features that appear on the antenna spectrum show a clear dip on top of the extinction and scattering spectrum in all cases, in accordance with our previous results. In the plasmonic absorption (blue lines), we observe the characteristic flip from a negative dip for dominantly absorbing antennas to a positive peak popping up on top of the absorption spectrum for the dominantly scattering antenna. In the case when the hosting antenna scattering equals its plasmonic absorption (middle panels in Figures $4 \mathrm{a}, \mathrm{b}$ ), the SEIRA contribution coming from the plasmonic absorption almost completely disappears, and the SEIRA signal is exclusively given by the plasmonic scattering mechanism.

We next evaluate the signal contrast for each situation (size of the dip or positive peak) caused by presence of the vibrational sample in the scattering, the absorption, and the resulting total extinction spectrum of the antenna-sample system. This signal contrast in the plasmonic cross section normalized to the vibrational sample volume is plotted for the antenna that is covered by the layer in Figure $4 c$ and for the sphere pair near the antenna in Figure $4 \mathrm{~d}$. This signal contrast is presented as a function of scattering-absorption ratio, similar to Figure 1c. In both types of samples the signal contrast in the extinction spectrum increases when the scattering contribution to the antenna's extinction increases (via enlarging the antenna volume) and then reaches a maximum for antennas that have comparable absorption and scattering $\left(\sigma_{\mathrm{abs}} \approx \sigma_{\mathrm{sca}}\right)$. The spectral contrast is therefore maximized in a region where it is dominantly given by the plasmonic scattering contribution. Interestingly, when the plasmonic scattering increases even more, the spectral contrast obtained in extinction again decreases. This behavior can be explained by splitting the extinction signal into the contributions from the antenna absorption and from scattering. The signal contrast given by the scattering (red dots) in the form of a dip grows when the antenna size is increased. If we increased the antenna size even more, the scattering signal contrast reaches a maximum for a certain antenna volume, and then it decreases, which is caused 
by an overall decrease of the associated local field acting on the sample. The dip-like SEIRA signal in extinction is further diminished because of the presence of the peak in absorption. A closer look at the data in Figure 4d reveals that in the case of an isolated vibrational nanoparticle coupled to a single plasmonic antenna the maximum of the extinction spectral contrast is slightly shifted toward antennas of larger volumes (larger proportions of scattering losses) with respect to those of layered samples in Figure 4c. To clarify this effect, we investigate the role of the sphere size on the overall trend of the spectral contrast (see Figure S6). We observed a shift of the spectral contrast maximum toward smaller antenna sizes when the size of the sample sphere was decreased, approaching the result obtained for the normalized signal contrast for antennas covered by a homogeneous layer of the vibrational material. A straightforward suggestion for the observation of SEIRA signals of samples that are themself almost invisible in the IR is thus to use antennas with $\sigma_{\mathrm{abs}} \approx \sigma_{\mathrm{sca}}$. The relatively flat peak maximum that appears in the extinction contrast as a function of scattering-absorption ratio suggests that a rather broad range of plasmonic scattering and absorption properties can provide similar SEIRA performance. With the antenna's scattering cross section at the plasmonic resonance comparable in magnitude to its absorption cross section, optimal SEIRA signals are obtained on the plasmonic background. Paradoxically, in such optimized situation the signal in SEIRA is almost exclusively given by the contribution of the modified plasmonic scattering. This result provides a robust rule that could be easily implemented in the experimental design of plasmonic antennas for SEIRA, as follows. For sensing of very small amounts of weak oscillator molecules, the near-field should be as large as possible. As commonly realized, this means positioning of the sample into the antenna's hot spot and tuning of the plasmonic resonance frequency (antenna plus sample) to the resonance frequency of the sample. Second, as we have investigated here for the linear IR antennas, tuning of the ratio of the antenna's scattering and absorption, so that $\sigma_{\mathrm{abs}} \approx \sigma_{\mathrm{sca}}$ leads to a superior SEIRA signal in the extinction cross section (the signal usually accessed in experiments). The control over the regime of the antenna's scattering performance could be easily achieved by tuning the antenna's geometrical dimensions, keeping its resonance frequency intact. The condition $\sigma_{\mathrm{abs}} \approx \sigma_{\mathrm{sca}}\left(\omega_{\mathrm{res}}^{2} T \approx \omega_{\tau}\right)$ can be checked, for example, via fitting the normalized extinction cross section to the analytical model eqs 1 and 2 and comparing the scattering maximum to the "ideal" value of $6 \pi c^{2} /\left(n_{\text {host }} \omega_{\text {res }}\right)^{2}$. The optimal ratio is $\approx 1 / 2$. If this condition is fulfilled, SEIRA signals, even from weakly IR-active biomolecules, can be measured best, since plasmonic extinction shows the maximum possible vibrational window resonance (dip).

We would like to mention here other plasmonic materials that have been used for SEIRA like for example graphene nanostructures. $^{37,38}$ Doped graphene nanoribbons with their monolayer thickness may show plasmonic absorption in the IR but do almost not scatter the light (i.e., $T \rightarrow 0$ ). In terms of our rule for maximum SEIRA enhancement, such plasmonic objects should have as low as possible intrinsic damping for SEIRA applications.

\section{SUMMARY AND CONCLUSION}

In summary, we have explored the role of plasmonic absorption and scattering on the formation of a measurable SEIRA signal, based on a numerical study of the infrared response of linear plasmonic antennas in the presence of samples from a material with IR-active vibrational oscillators. An optimal condition for the SEIRA signal is obtained when the hosting antenna absorption and scattering cross sections are very similar, emphasizing the paradoxical importance of antenna scattering in the generation of a strong SEIRA signal (usually considered as the enhanced vibrational absorption). In this situation, plasmonic scattering is the main contributor to the SEIRA spectral contrast that is measured in extinction spectra. A simple analytical model for the absorption and scattering cross section can be applied to design this optimal condition for the practical application in SEIRA experiments.

On the basis of the example of linear antennas, we established a simple condition for optimization of the vibrational signal in SEIRA via tuning the proportion between the antennas' intrinsic absorption losses and their scattering losses. Under consideration of the real intrinsic electronic relaxation rate, this ratio can be easily optimized by the adjustment of the geometrical antenna size and proportions (i.e., the volume and the aspect ratio of linear antennas). Our findings suggest that the antennas with an intrinsic damping comparable to radiation damping provide optimal SEIRA spectral contrast. It is strongly advisible to implement this simple condition for practical use in the experimental design of SEIRA experiments. The rule should be applicable in all cases where the analytical model eqs 1 and 2 reasonably describes the plasmonic spectrum. Furthermore, we assume that the rule allows the estimate of trends in the plasmonic performance for enhanced spectroscopies.

We also clarified that different regimes of antenna-sample coupling can be achieved in SEIRA, ranging from the regime of weak coupling for antennas covered by thin layers of molecules with weak vibrational response, to the strong coupling regime where the hosting antennas interact with strong collective phononic resonances of materials such as silicon oxide, for example.

\section{ASSOCIATED CONTENT}

\section{S Supporting Information}

The Supporting Information is available free of charge on the ACS Publications website at DOI: 10.1021/acs.jpcc.5b08344.

Estimate of an IR absorption cross section, image of a nanoantenna, influence of the intrinsic scattering rate and of the nanoantenna diameter on cross sections, detailed particle geometry and IR response, phonon signal in dependency on the particle position on the antenna (including the apex position in Figure 3a), and an additional (to Figure 4) set of simulations with a sphere of a diameter of only $15 \mathrm{~nm}$ (PDF)

\section{AUTHOR INFORMATION}

\section{Corresponding Author}

*E-mail pucci@kip.uni-heidelberg.de (A.P.).

\section{Author Contributions}

T.N., C.H., and J.V. contributed equally.

Notes

The authors declare no competing financial interest.

\section{ACKNOWLEDGMENTS}

J.V. and C.H. acknowledge financial support by the Heidelberg Graduate School of Fundamental Physics. C.H. acknowledges financial support by the Helmholtz Graduate School for Hadron and Ion Research. We acknowledge SOLEIL for 
provision of synchrotron radiation facilities, and we thank P. Dumas and F. Jamme for assistance in using the spectrometers at the beamline SMIS. We acknowledge the synchrotron light source ANKA for provision of instruments at their beamlines and thank D. Moss and M. Süpfle for their assistance in using beamline IR2. We are grateful for computational resources provided by the bwUniCluster of the federal state of BadenWürttemberg, Germany. We additionally thank X. Yin for sample preparation. J.A. and T.N. acknowledge the project FIS2013-14481-P from the Spanish Ministry of Economy and Competitiveness (MINECO), and ETORTEK project nanoGUNE'14 from the Department of Industry of the Basque Country Government. F.N. acknowledges financial support by the Baden-Württemberg-Stiftung (PROTEINSENS).

\section{REFERENCES}

(1) Bradshaw, A.; Pritchard, J. Infra-Red Spectra of Carbon Monoxide on Evaporated Nickel Films. Surf. Sci. 1969, 17, 372-386.

(2) Griffiths, P. R. Spectroscopic Properties of Inorganic and Organometallic Compounds: Techniques, Materials and Applications; Royal Society of Chemistry: Cambridge, 2013; Vol. 44, pp 95-122.

(3) Persson, B.; Liebsch, A. Optical Properties of Two-Dimensional Systems of Randomly Distributed Particles. Phys. Rev. B 1983, 28, 4247-4254.

(4) Priebe, A.; Sinther, M.; Fahsold, G.; Pucci, A. The Correlation between Film Thickness and Adsorbate Line Shape in Surface Enhanced Infrared Absorption. J. Chem. Phys. 2003, 119, 4887-4890.

(5) Neubrech, F.; Pucci, A.; Cornelius, T. W.; Karim, S.; GarcáEtxarri, A.; Aizpurua, J. Resonant Plasmonic and Vibrational Coupling in a Tailored Nanoantenna for Infrared Detection. Phys. Rev. Lett. 2008, 101, 157403.

(6) Neubrech, F.; Pucci, A. Plasmonic Enhancement of Vibrational Excitations in the Infrared. IEEE J. Sel. Top. Quantum Electron. 2013, 19, 4600809.

(7) Brown, L. V.; Zhao, K.; King, N.; Sobhani, H.; Nordlander, P.; Halas, N. J. Surface-Enhanced Infrared Absorption Using Individual Cross Antennas Tailored to Chemical Moieties. J. Am. Chem. Soc. 2013, 135, 3688-3695.

(8) Hoffmann, J. M.; Yin, X.; Richter, J.; Hartung, A.; Maß, T. W. W.; Taubner, T. Low-Cost Infrared Resonant Structures for SurfaceEnhanced Infrared Absorption Spectroscopy in the Fingerprint Region from 3 to $13 \mu \mathrm{m}$. J. Phys. Chem. C 2013, 117, 11311-11316.

(9) Chen, K.; Adato, R; Altug, H. Dual-Band Perfect Absorber for Multispectral Plasmon-Enhanced Infrared Spectroscopy. ACS Nano 2012, 6, 7998-8006.

(10) D'Andrea, C.; Bochterle, J.; Toma, A.; Huck, C.; Neubrech, F.; Messina, E.; Fazio, B.; Maragò, O. M.; Di Fabrizio, E.; Lamy; de La Chapelle, M.; et al. Optical Nanoantennas for Multiband SurfaceEnhanced Infrared and Raman Spectroscopy. ACS Nano 2013, 7, 3522-3531.

(11) Huck, C.; Neubrech, F.; Vogt, J.; Toma, A.; Gerbert, D.; Katzmann, J.; Härtling, T.; Pucci, A. Surface-Enhanced Infrared Spectroscopy Using Nanometer-Sized Gaps. ACS Nano 2014, 8, 4908-4914.

(12) Wang, T.; Nguyen, V. H.; Buchenauer, A.; Schnakenberg, U.; Taubner, T. Surface Enhanced Infrared Spectroscopy with Gold Strip Gratings. Opt. Express 2013, 21, 9005-9010.

(13) Brown, L. V.; Yang, X.; Zhao, K.; Zheng, B. Y.; Nordlander, P.; Halas, N. J. Fan-Shaped Gold Nanoantennas above Reflective Substrates for Surface-Enhanced Infrared Absorption (SEIRA). Nano Lett. 2015, 15, 1272-1280.

(14) Huck, C.; Toma, A.; Neubrech, F.; Chirumamilla, M.; Vogt, J.; De Angelis, F.; Pucci, A. Gold Nanoantennas on a Pedestal for Plasmonic Enhancement in the Infrared. ACS Photonics 2015, 2, 497505 .
(15) Adato, R.; Altug, H. In-Situ Ultra-Sensitive Infrared Absorption Spectroscopy of Biomolecule Interactions in Real Time with Plasmonic Nanoantennas. Nat. Commun. 2013, 4, 2154.

(16) Adato, R.; Yanik, A. A.; Amsden, J. J.; Kaplan, D. L.; Omenetto, F. G.; Hong, M. K.; Erramilli, S.; Altug, H. Ultra-Sensitive Vibrational Spectroscopy of Protein Monolayers with Plasmonic Nanoantenna Arrays. Proc. Natl. Acad. Sci. U. S. A. 2009, 106, 19227-19232.

(17) Neubrech, F.; Weber, D.; Enders, D.; Nagao, T.; Pucci, A. Antenna Sensing of Surface Phonon Polaritons. J. Phys. Chem. C 2010, 114, 7299-7301.

(18) Weber, D.; Albella, P.; Alonso-González, P.; Neubrech, F.; Gui, H.; Nagao, T.; Hillenbrand, R.; Aizpurua, J.; Pucci, A. Longitudinal and transverse coupling in infrared gold nanoantenna arrays: long range versus short range interaction regimes. Opt. Express 2011, 19, 1504715061.

(19) Alonso-González, P.; Albella, P.; Neubrech, F.; Huck, C.; Chen, J.; Golmar, F.; Casanova, F.; Hueso, L. E.; Pucci, A.; Aizpurua, J.; Hillenbrand, R. Experimental Verification of the Spectral Shift between Near- and Far-Field Peak Intensities of Plasmonic Infrared Nanoantennas. Phys. Rev. Lett. 2013, 110, 203902.

(20) Vogt, J.; Huck, C.; Neubrech, F.; Toma, A.; Gerbert, D.; Pucci, A. Impact of the Plasmonic Near- and Far-Field Resonance-Energy Shift on the Enhancement of Infrared Vibrational Signals. Phys. Chem. Chem. Phys. 2015, 17, 21169-21175.

(21) Eilers, P. H. C. A Perfect Smoother. Anal. Chem. 2003, 75, $3631-3636$

(22) Kats, M. A.; Yu, N.; Genevet, P.; Gaburro, Z.; Capasso, F. Effect of Radiation Damping on the Spectral Response of Plasmonic Components. Opt. Express 2011, 19, 21748-21753.

(23) Doyle, W. T. Electrodynamic Response of Metal Spheres. J. Opt. Soc. Am. A 1985, 2, 1031-1034.

(24) Pelton, M.; Bryant, G. Introduction to Metal-Nanoparticle Plasmonics; A Wiley-Science Wise Co-Publication; Wiley: New York, 2013.

(25) Palik, E. D. Handbook of Optical Constants of Solids; Academic Press: New York, 1985.

(26) Trollmann, J.; Pucci, A. Infrared Dielectric Function of Gold Films in Relation to Their Morphology. J. Phys. Chem. C 2014, 118, 15011-15018.

(27) Seok, T. J.; Jamshidi, A.; Kim, M.; Dhuey, S.; Lakhani, A.; Choo, H.; Schuck, P. J.; Cabrini, S.; Schwartzberg, A. M.; Bokor, J.; Yablonovitch, E.; Wu, M. C. Radiation Engineering of Optical Antennas for Maximum Field Enhancement. Nano Lett. 2011, 11, $2606-2610$

(28) Gunde, M. K. Vibrational Modes in Amorphous Silicon Dioxide. Phys. B 2000, 292, 286-295.

(29) Berreman, D. Infrared Absorption at Longitudinal Optic Frequency in Cubic Crystal Films. Phys. Rev. 1963, 130, 2193-2198.

(30) Kliewer, K. L.; Fuchs, R. Optical Modes of Vibration in an Ionic Crystal Slab Including Retardation. I. Nonradiative Region. Phys. Rev. 1966, 144, 495-503.

(31) Senet, P.; Lambin, P.; Lucas, A. Standing-Wave Optical Phonons Confined in Ultrathin Overlayers of Ionic Materials. Phys. Rev. Lett. 1995, 74, 570-573.

(32) Tassin, P.; Zhang, L.; Zhao, R.; Jain, A.; Koschny, T.; Soukoulis, C. M. Electromagnetically Induced Transparency and Absorption in Metamaterials: The Radiating Two-Oscillator Model and Its Experimental Confirmation. Phys. Rev. Lett. 2012, 109, 187401.

(33) Adato, R; Artar, A.; Erramilli, S.; Altug, H. Engineered Absorption Enhancement and Induced Transparency in Coupled Molecular and Plasmonic Resonator Systems. Nano Lett. 2013, 13, 2584-2591.

(34) Alonso-Gonzalez, P.; Albella, P.; Schnell, M.; Chen, J.; Huth, F.; Garcia-Etxarri, A.; Casanova, F.; Golmar, F.; Arzubiaga, L.; Hueso, L.; et al. Resolving the Electromagnetic Mechanism of Surface-Enhanced Light Scattering at Single Hot Spots. Nat. Commun. 2012, 3, 684.

(35) Dregely, D.; Neubrech, F.; Duan, H.; Vogelgesang, R.; Giessen, H. Vibrational Near-Field Mapping of Planar and Buried ThreeDimensional Plasmonic Nanostructures. Nat. Commun. 2013, 4, 2237. 
(36) Amenabar, I.; Poly, S.; Nuansing, W.; Hubrich, E. H.; Govyadinov, A. A.; Huth, F.; Krutokhvostov, R.; Zhang, L.; Knez, M.; Heberle, J.; et al. Structural Analysis and Mapping of Individual Protein Complexes by Infrared Nanospectroscopy. Nat. Commun. 2013, 4, 2890.

(37) Li, Y.; Yan, H.; Farmer, D. B.; Meng, X.; Zhu, W.; Osgood, R. M.; Heinz, T. F.; Avouris, P. Graphene Plasmon Enhanced Vibrational Sensing of Surface-Adsorbed Layers. Nano Lett. 2014, 14, 1573-1577.

(38) Rodrigo, D.; Limaj, O.; Janner, D.; Etezadi, D.; García de Abajo, F. J.; Pruneri, V.; Altug, H. Mid-Infrared Plasmonic Biosensing with Graphene. Science 2015, 349, 165-168. 